\title{
Fluorescence NanoParticle Detection in a liquid sample using the Smartphone for Biomedical application
}

Anand G ( $\sim$ g.anand777@gmail.com )

Madras Institute of Technology https://orcid.org/0000-0001-6403-5577

Thyagarajan $\mathrm{T}$

Madras Institute of Technology

Sabitha Ramakrishnan

Madras Institute of Technology

\section{Research Article}

Keywords: Android, Fluorescence, Nanoparticle, Pathogens, Smartphone

Posted Date: July 7th, 2021

DOl: https://doi.org/10.21203/rs.3.rs-616931/v1

License: (c) (i) This work is licensed under a Creative Commons Attribution 4.0 International License.

Read Full License 


\title{
Fluorescence NanoParticle Detection in a liquid sample using the Smartphone for Biomedical application
}

\author{
Anand. $G^{1}$, T. Thyagarajan ${ }^{1}$, Sabitha Ramakrishnan ${ }^{1}$
}

\begin{abstract}
In the present work, a Smartphone-based Fluorescence Nanoparticle Detector (SPF-NPD) was developed. This method is intended for use in the identification of biological agents in biomedical applications. Here, an android application-based algorithm was developed to analyze the fluorescent nanoparticle intensity level in a target sample. The setup consists of an LED light source, an Eppendorf tube holder, and a smartphone to acquire the fluorescent intensity level in the sample to enable the detection of pathogens within few seconds. High-resolution cameras available on recent smartphones have made live detection more accurate and convenient for healthcare applications. The concept of fluorescent nanoparticle detection with a smartphone has led to a portable device and having potential application in healthcare. In this proposed method the intensity level is analyzed with 5 pixels algorithm, the center pixel followed by four immediate neighbours' pixels which can analyze with minimal sample quantity. Also, the robustness of the developed algorithm was verified with various megapixel camera ranges from 8 MP to 20 MP.
\end{abstract}

Keywords: Android, Fluorescence, Nanoparticle, Pathogens, Smartphone.

\section{INTRODUCTION}

Android is now the most used mobile operating system in the world. It has now more users, worldwide than any other mobile operating system (1). Android plays a successful role in the medical field $(2,3)$. Human beings wherever they reside are hemmed in by a briny of pathogens. These pathogens can be in the form of fungi, bacteria, viruses, and many other microorganisms (4). They reside in every niche of the environment. Many of these microbes survive without instigating any damage to the body, while some conquer our body-producing disease (5)(3). The microorganism growth may take place at a faster and hence the need for instantaneous detection of pathogenic agents has increased tremendously $(6,7)$.

In the medical field, the detection of pathogenic bacteria in food, water, and air in the shortest time has been an important aspect for researchers. Traditional strategies of microorganism detection need analytical laboratories, typically in centralized facilities, which need substantial capital and extremely skilled manpower (8).

Although these achieve sensitive and selective bacterial detection (9), they require a longer time to yield a result (10). Fluorescence detection is well-suited for the detection of pathogenic contamination having high sensitivity, minimal time necessity for assessment, and lack of sample contact (11). To overcome this, a new system is designed, which uses the principle of fluorescent nanoparticle detection (12) to identify the cellular and molecular components of each sample with a high amount of specificity and to examine impurities and disease conditions (5)(13).

Anand G

g.anand777@gmail.com

1. Department of Instrumentation Engineering, Madras Institute of Technology, Anna University, Chennai, India
Among the best software detection methods, the Android-based mobile application detection system has now commanded its application to infectious disease diagnostics (14)(15). In this paper, we created an android mobile application to analyze the intensity of the fluorescence to make a quantitative analysis of the target sample. In this proposed method the intensity level is analyzed with 5 pixels, center pixel followed by four immediate neighbors' pixels which analyzes with minimal sample quantity (16)(17).

\section{FluORESCENT DETECTION TECHNIQUE}

Fluorescence is the absorption of light energy by a molecule at one wavelength and its reemission at another wavelength (5). Some amount of energy is dissipated as heat when electrons return to their ground state (18)(19). Hence, the light produced always has an elongated wavelength than the engrossed light due to limited energy loss by the molecule before radiation (20). Fluorescence is a three-stage procedure that usually ensues in particular molecules called fluorophores or fluorescent dyes (21) (22). A fluorescent nanoparticle is a fluorophore designed to focus within a specific region of a biological agent and to retort to a specific stimulus (10)(3).

The process responsible for the fluorescent nanoparticles is elucidated (23) by the simple electronic state diagram shown in figure 1.

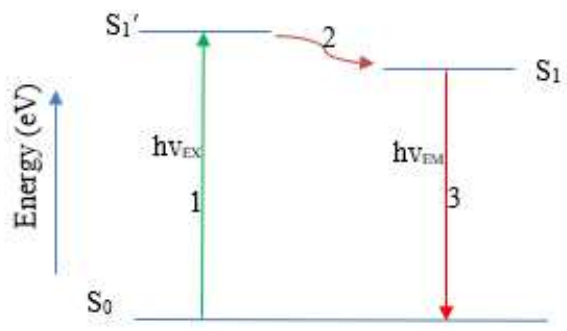

Fig. 1 Photon electronic state diagram

When photon energy is stimulated by an external light source such as a Light Emitting Diode (LED), fluorophore 
absorbs its energy creating an excited electronic singlet state $\left(\mathrm{S}_{1}{ }^{\prime}\right)$. The energy of $\mathrm{S}_{1}{ }^{\prime}$ is partially dissipated as heat, yielding a relaxed singlet excited state $\left(S_{1}\right)(24)$. After some time, fluorophore returns to the ground state $\left(\mathrm{S}_{0}\right)$ emitting a photon having a longer wavelength and lower energy than the excitation energy due to thermal and vibrational losses during the transient exciting lifetime.

The quantity of wavelength of the emitted energy depends on each fluorophore and the chemical setting of the fluorophore (25)(26). The deterioration of fluorescence intensity as a function of time in a uniform population of molecules excited with a brief pulse of light is described by an exponential function as given in equation (1).

$$
I(t)=I(o) \cdot e^{(-t / T)} \ldots \ldots(1)
$$

Where $I(t)$ is the fluorescence intensity measured at time $t$, $\mathrm{I}(\mathrm{o})$ is the initial intensity observed immediately after excitation and $\mathrm{T}$ is the fluorescence lifetime.

\section{A. Stokes's shift}

Stokes shift is the difference in energy or wavelength between states of the band maxima of the absorption and emission spectra of the same electronic transition (2) as shown in figure 2. Usually, fluorophore having a higher stokes shift is preferred.

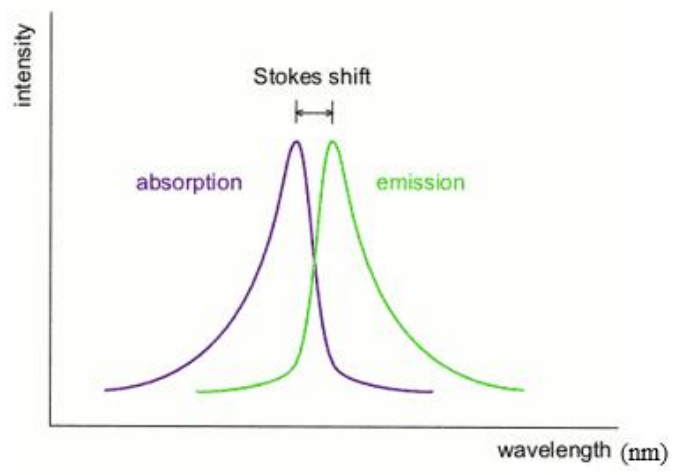

Fig. 2. Stokes's shift (Excitation and emission spectral response)

\section{MATERIAls AND Materials}

A fluorescence-based system consists of three main components namely: 1) A sample holder to contain the fluorophore for investigation, 2) An excitation source to stimulate the fluorophore, and 3) A smartphone-based mobile application to analyze the target sample. The basic functional block diagram of the android-based mobile application for fluorescence-based nanoparticle detection system is shown in the figure.

It is an additive color model in which red, green, and blue are added together to form a broad range of colors. The HSV is also known as HSB. Using this model, an object color can be identified, and it helps to prevent the influence of light intensity from the outside. In fig. 3, the excitation source from LED is used to stimulate the fluorophore (Fluorescent bio-sample). The exciting light source will be absorbed by fluorophores and light energy of another wavelength will be emitted. Further, light energy will be captured and used for performing intensity analysis using a smartphone.

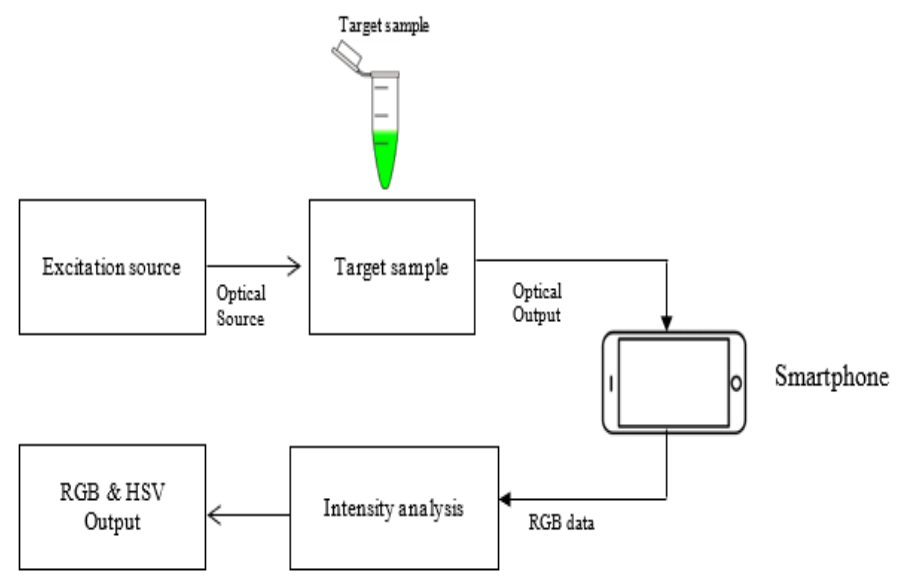

RGB: Red, Green, Blue; HSV: Hue, Saturation, Value; HSB: Hue, Saturation, Brightness Fig. 3. Block diagram of the SPF-NPD

\section{A. Excitation source}

The illumination system consists of an excitation source i.e., LEDs with required optical components. The light source provides the energy that excites the samples by emitting light. Fluorophores are molecules that absorb light at one spectrum and emit at a higher spectrum with lower energy compared to excitation energy. This property is suitable for detecting when the absorption or emission process is influenced by analytes of interest(27).

To identify fluorescence with high accuracy, the excitation light should be distinct from the emission light. Hence, the source can be any device that emits radiation having wavelengths between 450 and 495 nanometers i.e., blue wavelength region. An example of such a suitable source includes blue color LED having that wavelength emission. The spectrum of the excitation source should not include the emission range. White LED is used as a source to provide a light of wavelength $494 \mathrm{~nm}$ with uniform illumination across the area. The operating characteristics of LED are presented in Table-1.

Table 1. Operating characteristics for LED

\begin{tabular}{l|l}
\hline Parameter & Value \\
\hline Product ID & L1-0-b5th15-1 \\
Angle & 15 \\
Package & $5 \mathrm{~mm}$ \\
Peak wavelength & $470 \mathrm{~nm}$ \\
Luminous intensity & $3460 \mathrm{mcd}$ type. @ 20mA \\
Max Forward current & $30 \mathrm{Ma}$ \\
Forward voltage & $3.6 \mathrm{~V}$ typ.4.0V max @20mA \\
Max reverse current & $50 \mathrm{uA} @ 5 \mathrm{~V}$ \\
Max reverse voltage & $5 \mathrm{~V}$ \\
Power dissipation & $120 \mathrm{Mw}$ \\
Operating temperature & -30 to $+85 \mathrm{C}$ \\
\hline
\end{tabular}




\section{B. Sample Preparation}

Fluorescent is the dye that is currently used for testing both instruments for initial screening. The dye used for live sampling is DiOC18. This is because an enzyme produced by microscopic organisms helps to break the bonds in these dyes, thus exposing the fluorescing part of the compound (28). Hence, as a proof of concept, a fluorescent dye is used for detection for prototype development and testing without biological organisms. The molecular weight of fluorescent is 376.3g. Molarity is calculated by using the formula given in equation (2)

$$
\text { Molarity }=\text { Grams } /(\text { Molecular Weight } * \text { Volume }) \ldots
$$

To prepare a $1 \mathrm{ml}$ quantity of $1 \mathrm{M}$ (Molar) concentration) the solution, $3.763 \mathrm{~g}$ of DioC18 is required. Based on equation 2 for $1 \mathrm{ml}$ quantity of $10 \mathrm{mM}$ concentration solution, $3.763 \mathrm{mg}$ of DioC18 is required. Using a weighing machine DioC18 is measured and added with distilled water and then placed in a micro-centrifuge tube as a reference stock solution. To prepare the working solution, serial dilution is done from the stock solution. To prepare $1 \mathrm{ml}$ of $1 \mathrm{mM}$ solution, $100 \mu \mathrm{l}$ of the stock is dissolved in $900 \mu \mathrm{l}$ of distilled water. Further, serial dilution can be done for various sample concentrations as per the flowchart given in Figure 4.

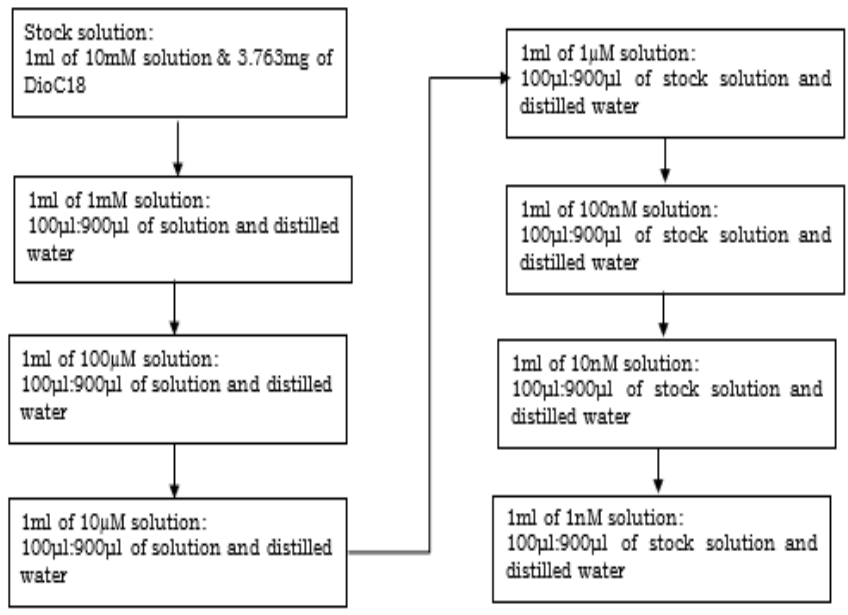

Fig 4. Sample preparation and serial dilution

\section{Smartphone}

In the proposed work, the smartphone is used as a detector to determine the fluorescent nanoparticle's intensity level(29). The minimum required configuration for a smartphone as a detector is above the Jellybean android version and the camera must be 8 megapixels (MP) and above. Various android smartphone phones were used to analyze and determine the efficiency of the algorithm and robustness of the developed android application.

\section{Experimental Setup}

Figure 5 shows the experimental setup of the SPFND method which includes the light source and sample holder. A refers to the dark enclosure for the excitation light source and sample holder. B indicates the camera's open slit to capture the intensity level. It has a slit to capture the intensity level of the sample using the smartphone as shown in figure 6. C and D refer to the RGB and HSV values respectively.

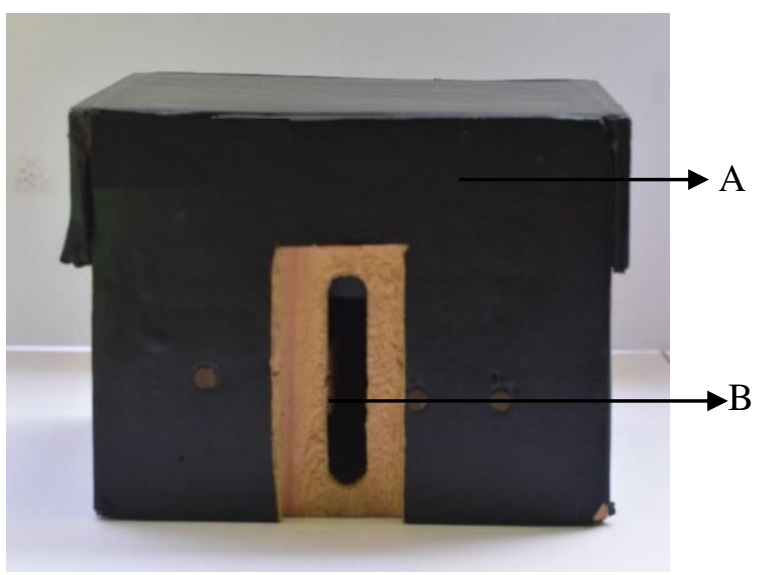

Fig. 5. Sample enclosure box

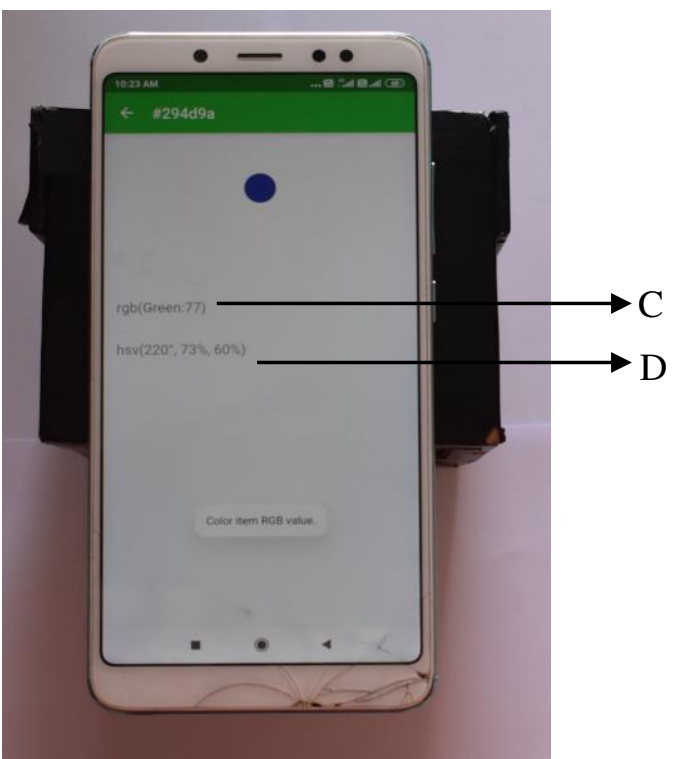

Fig. 6. Experimental setup with smartphone

\section{E. Software Development}

This intensity is based on the primary color values of the fluorescence. The smartphone algorithm is developed in the android platform to analyze the intensity. The setup equipped with a fluorescent (DiOC18) sample is attached to the mobile.

The application was created in Android studio platform version 1.3.1 using the java programming language. The Application programming interface (API) level of this application started from API 17 (Jellybean). This application detects the color which was emitted by fluorescein dye. The exciting light source passes through the sample where the fluorescein will absorb the light source. The mobile application created detects the intensity of the fluorescence sample and gives the RGB values and HSV (Hue, Saturation, 
and Value) values of the respective samples. The color of the respective sample is detected by the width and height of the pointer radius. Here, we fixed the pointer radius as 5 in pixels. The RGB values can be found out using the YUV420 conversion process.

The default conversion process is as follows, size. total $=$ size. width $*$ size. height.

$$
\begin{aligned}
& \mathrm{y}=\operatorname{yuv}[\text { position. } \mathrm{y} * \text { size. width }+ \text { position. } \mathrm{x}] \ldots \text { (4) } \\
& \mathrm{u}=\operatorname{yuv}\left[\left(\operatorname{position} \cdot \frac{\mathrm{y}}{2}\right) *\left(\operatorname{size} \cdot \frac{\text { width }}{2}\right)+\left(\text { position } \cdot \frac{\mathrm{x}}{2}\right)+\right. \\
& \text { size. total] ...... (5) } \\
& \mathrm{v}=\operatorname{yuv}\left[\left(\text { position. } \frac{\mathrm{y}}{2}\right) *\left(\text { size. } \frac{\text { width }}{2}\right)+\left(\text { position } \cdot \frac{\mathrm{x}}{2}\right)+\right. \\
& \text { size. total } \left.+\left(\operatorname{size} \cdot \frac{\text { total }}{4}\right)\right] \\
& \mathrm{rgb}=\mathrm{Y}^{\prime} \mathrm{UV} \text { 444toRGB888(y, u, v) }
\end{aligned}
$$

This format is the standard image format on the Android camera preview. The $\mathrm{Y}^{\prime} \mathrm{UV}$ model defines a color space in terms of one luma component $\left(\mathrm{Y}^{\prime}\right)$ and two chrominance components, called $\mathrm{U}$ and $\mathrm{V}$ respectively. Luminance is denoted by $Y$. The prime symbols (') denote gamma correction, with "luminance" meaning physical linear-space brightness, while "luma" is (nonlinear) perceptual brightness. The developed algorithm gives the RGB value of the respective image and displays the green component of that image. From the RGB value, we can get the HSV value. The mathematical relationship for RGB to HSV conversion is given in equations 8 to 9 .

Hue calculation:

$$
\mathrm{H}=\left\{\begin{array}{l}
60^{\circ} \times\left(\frac{\mathrm{G}^{\prime}-\mathrm{B}^{\prime}}{\Delta} \bmod 6\right), \mathrm{C}_{\max }=\mathrm{R}^{\prime} \\
60^{\circ} \times\left(\frac{\mathrm{B}^{\prime}-\mathrm{R}^{\prime}}{\Delta} \bmod 6\right), \mathrm{C}_{\max }=\mathrm{G}^{\prime} \ldots \ldots(8) \\
60^{\circ} \times\left(\frac{\mathrm{R}^{\prime}-\mathrm{G}^{\prime}}{\Delta} \bmod 6\right), \mathrm{C}_{\max }=\mathrm{B}^{\prime}
\end{array}\right.
$$

Saturation calculation:

$$
\mathrm{S}= \begin{cases}0, & \mathrm{C}_{\max }=0 \\ \Delta, & \mathrm{C}_{\max } \neq 0 \\ \mathrm{C}_{\max }\end{cases}
$$

Value calculation:

$$
\mathrm{V}=\mathrm{Cmax}
$$

Here, R' represents R/255. Similarly, G' represents $G / 255$, and $B^{\prime}$ represents $B / 255$. The maximum and minimum values are given as follows: $C_{\max }=\max \left(R^{\prime}, G^{\prime}, B^{\prime}\right) C_{\min }=\min \left(R^{\prime}\right.$, $\left.\mathrm{G}^{\prime}, \mathrm{B}^{\prime}\right), \Delta=\mathrm{C}_{\max }-\mathrm{C}_{\min }$.

\section{RESULT AND DISCUSSION}

When the sample is excited with the light source, the sample undergoes the Stokes shift phenomenon. Later, the intensity level of the sample was captured and measured using a smartphone application. After capturing the image, to measure the sample intensity, the developed android algorithm extracts the RGB and HSV values. For the fluorescence count, $\mathrm{G}$ value intensity was considered. Based on the calibration, the corresponding RGB and HSV values will give the number of fluorescent particles present.

The calibration chart is shown in figure 7. From figure 7 as the concentration of the sample increases, the RGB value in pixels also increases. At a higher concentration of $1000 \mathrm{nM}$, the RGB values attain their saturation. The calibration of RGB data with the sample concentration is performed and the active range is identified.

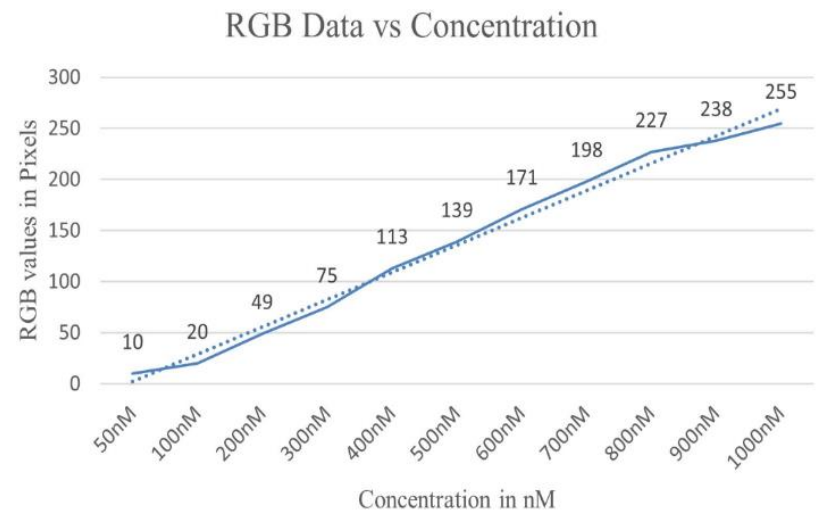

Fig. 7 Calibration chart for RGB vs known Sample Concentration

The result view page of the android application is shown in figure 8 . From figure 8 , it is observed that the amount of green value is equal to the intensity level of fluorescence present in the sample. For various levels of concentrations, equivalent amounts of green values will be produced and captured. It is also observed that the higher the concentration level, the higher is the intensity level i.e., higher green value.

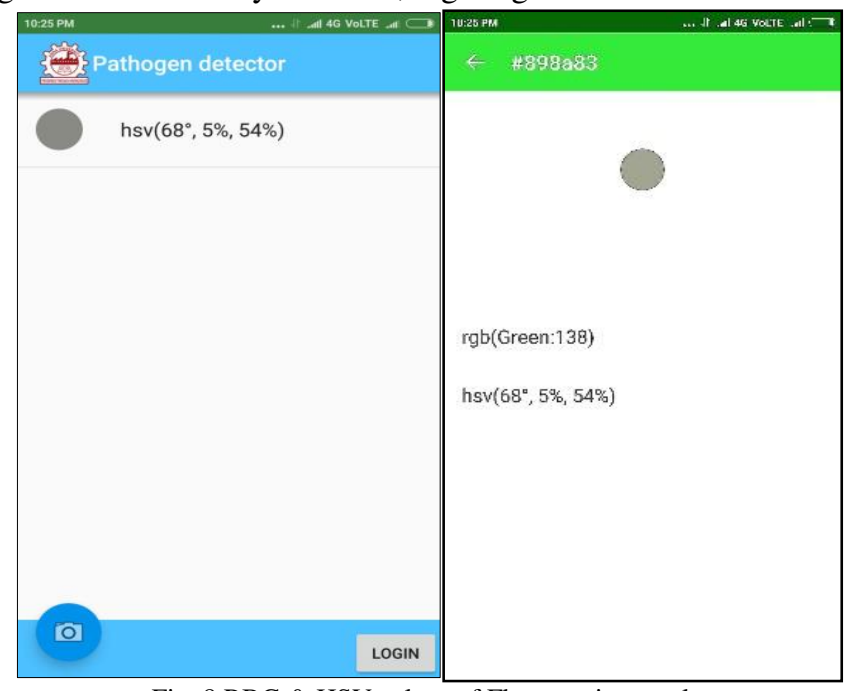

Fig. 8 RBG \& HSV values of Fluorescein sample

The sensitivity of SPF-NPD is compared with conventional devices namely: Perkin Elmer enspire and Picofluor. From 
figures 9 and 10 , it is observed that the sensitivities of Enspire and SPF-NPD are the same. It is also observed that the sensitivities of Picofluor and SPF-NPD are almost the same.

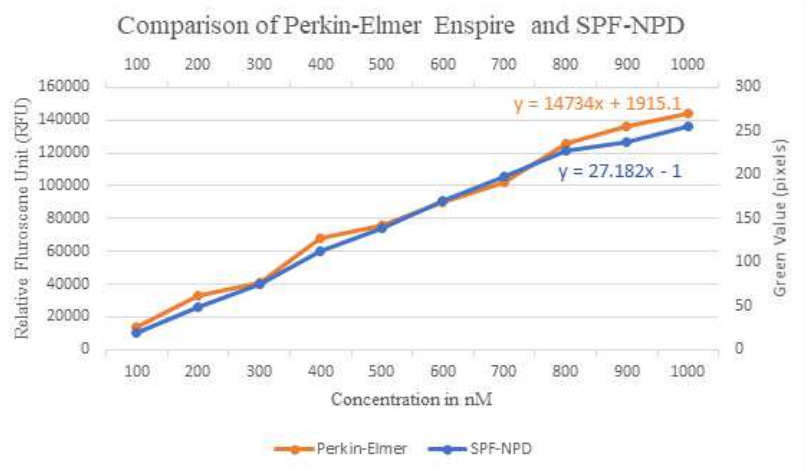

Fig. 9 Comparison of Perkin-Elmer Enspire and SPF-NPD

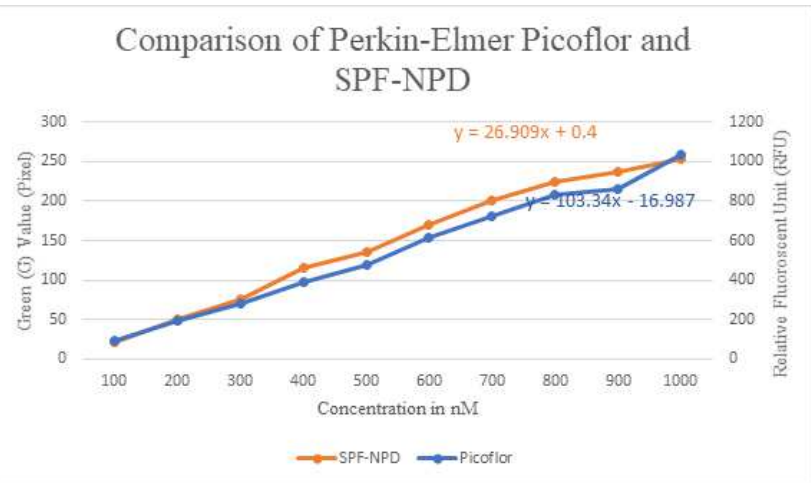

Fig. 10 Comparison of Perkin-Elmer Picoflor and SPF-NPD

The Robustness of the SPF-NPD algorithm with various smartphones (different camera MP) is shown in Figure 11. From figure 11, it is observed that the results obtained using various smartphones (different camera resolutions) remain equal.

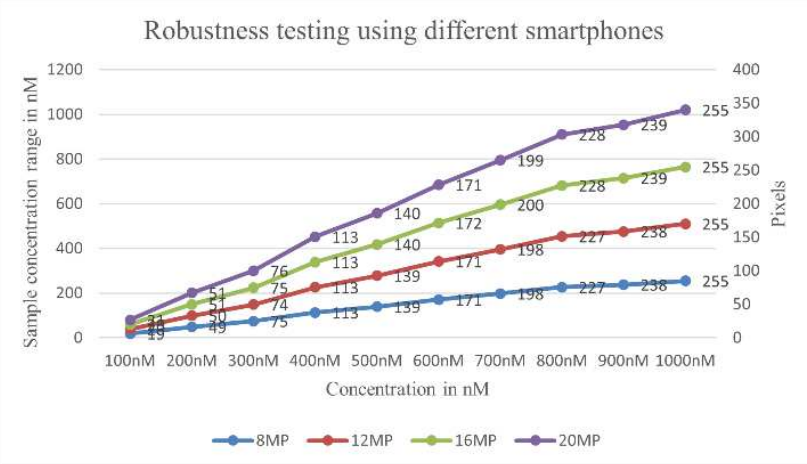

Fig. 11 Robustness of SPF-NPD algorithm with various smartphones (different camera MP)

This developed android app can be installed with any kind of smartphone which has the basic configuration has specified earlier. This proves the robustness and effectiveness of the developed algorithm. Also, comparatively, the proposed method is much cost-effective, portable, and easy to use.

\section{Conclusion}

A novel method for fluorescence detection is proposed for the detection of fluorescence nanoparticles using a smartphone in an android platform with the lowest concentration level of $100 \mathrm{nM}$. The intrinsic fluorescence detection method is deployed for the detection of nanoparticles without the necessity for sample contact. Here, a fluorescent dye is used as a nanoparticle. The fluorescent nanoparticle absorbs the light (485 $\mathrm{nm}$ wavelength) from the excitation source and emitted a particular wavelength $(515 \mathrm{~nm})$. This emitted light is captured using a smartphone to determine the intensity level and hence the nanoparticle count. From the comparison of sensitivity analysis, it is observed that the sensitivities of the prototype developed, and the conventional methods are almost equal. The performance of the prototype was also analyzed with various fluorescence concentration samples (without pathogen). The developed method saturates at $1000 \mathrm{nM}$ concentration. Also, the robustness of the developed application was tested with various camera resolutions ranging from $8 \mathrm{MP}$ to $20 \mathrm{MP}$.

\section{Author's Declarations}

-Funding- Not Applicable

-Conflicts of interest/Competing interests- Not Applicable

-Ethics approval/declarations- Not Applicable

-Consent to participate- Not Applicable

-Consent for publication- Not Applicable

-Availability of data and material/ Data availability- The datasets generated during the current study are available from the corresponding author on reasonable request.

-Code availability- Not Applicable

-Authors' contributions- Anand. G, Thyagarajan. T, Sabitha Ramakrishnan have equally contributed.

\section{REFERENCE}

1. Rezazadeh M, Seidi S, Lid M, Pedersen-Bjergaard S, Yamini Y. The modern role of smartphones in analytical chemistry. TrAC - Trends Anal Chem [Internet]. 2019;118:548-55. Available from: https://doi.org/10.1016/j.trac.2019.06.019

2. HESAM SHAHIN RW. Mobile phone as a fluorescence reader. Opt Appl. 2013;XLIII(3).

3. Rajendran VK, Bakthavathsalam P, Mohammed B, Ali J. Smartphone based bacterial detection using biofunctionalized fluorescent nanoparticles. Microchim Acta. 2014; 
4. Müller V, Sousa JM, Ceylan Koydemir H, Veli M, 14 Tseng D, Cerqueira L, et al. Identification of pathogenic bacteria in complex samples using a smartphone based fluorescence microscope. RSC Adv. 2018;8(64):36493502.

5. Wei Q, Qi H, Luo W, Tseng D, Ki SJ, Wan Z, et al. Fluorescent imaging of single nanoparticles and viruses on a smart phone. ACS Nano. 2013;7(10):9147-55.

6. Rong Z, Wang Q, Sun N, Jia X, Wang K, Xiao R, et al. Smartphone-based fluorescent lateral flow immunoassay platform for highly sensitive point-of-care detection of Zika virus nonstructural protein 1. Anal Chim Acta [Internet]. 2019;1055:140-7. Available from: https://doi.org/10.1016/j.aca.2018.12.043

7. Shrivastava S, Lee W Il, Lee NE. Culture-free, highly sensitive, quantitative detection of bacteria from minimally processed samples using fluorescence imaging by smartphone. Biosens Bioelectron [Internet]. 2018;109:90-7. Available from: https://doi.org/10.1016/j.bios.2018.03.006

8. Xu D, Huang X, Guo J, Ma X. Automatic smartphonebased microfluidic biosensor system at the point of care. Biosens Bioelectron [Internet]. 2018;110:78-88. Available https://doi.org/10.1016/j.bios.2018.03.018

9. Wei Q, Acuna G, Kim S, Vietz C, Tseng D, Chae J, et al. Plasmonics enhanced smartphone fluorescence microscopy. Sci Rep. 2017;7(1):1-10.

10. Hanson GT, Hanson BJ. Fluorescent Probes for Cellular Assays. 2008;505-13.

11. Urdea AM, Penny LA, Olmsted SS, Giovanni MY, Kaspar P, Shepherd A, et al. Requirements for high impact diagnostics in the developing world.

12. Xianyu Y, Wang Q, Chen Y. Magnetic particles-enabled biosensors for point-of-care testing. TrAC - Trends Anal 22. Chem [Internet]. 2018;106:213-24. Available from: https://doi.org/10.1016/j.trac.2018.07.010

13. Xu X, Akay A, Wei H, Wang S, Pingguan-Murphy B, Erlandsson BE, et al. Advances in Smartphone-Based 23. Point-of-Care Diagnostics. Proc IEEE. 2015;103(2):236-47.
Mudanyali O, Navruz I. Smart Rapid Diagnostics Test Reader running on a Cell-Phone for Real-time Mapping of Epidemics. 2012;

15. Wenhao Zhaoa, Shulin Tiana, Lei Huanga, Jinhong Guob, Ke Liua and LD. Smartphone-based Biomedical sensory system. Analyst. 2020;(DOI: 10.1039/C9AN02294E).

16. Singh S, Halder A, Sinha O, Sarkar PK, Singh P, Banerjee A, et al. Nanoparticle-based "turn-on" scattering and post-sample fluorescence for ultrasensitive detection of water pollution in wider window. PLoS One. 2020;15(1).

17. Zheng Q, Wu H, Jiang H, Yang J, Gao Y. Development of a smartphone-based fluorescent immunochromatographic assay strip reader. Sensors (Switzerland). 2020;20(16):1-12.

18. Liu J, Geng Z, Fan Z, Liu J, Chen H. Point-of-care testing based on smartphone: The current state-of-the-art (20172018). Biosens Bioelectron [Internet]. 2019;132(November 2018):17-37. Available from: https://doi.org/10.1016/j.bios.2019.01.068

19. Sharma J, Ono T, Yukino R, Miyashita H, Hanyu N, Handa $\mathrm{H}$, et al. Smartphone based platform for real-time sharing of medical diagnostics information by optical detection of functionalized fluorescent magnetic nanoparticles. Biomed Phys Eng Express. 2019;5(3).

20. Zhu H, Mavandadi S, Coskun AF, Yaglidere O, Ozcan A. Optofluidic Fluorescent Imaging Cytometry on a Cell Phone. 2011;6641-7.

21. Jaffrezic-Renault N, Errachid A. Analytical microsystems for biomedical and environmental applications. Biocybern Biomed Eng [Internet]. 2011;31(4):3-16. Available from: http://dx.doi.org/10.1016/S02085216(11)70022-5

Coskun AF, Ozcan A. Computational imaging, sensing and diagnostics for global health applications. Curr Opin Biotechnol [Internet]. 2014;25:8-16. Available from: http://dx.doi.org/10.1016/j.copbio.2013.08.008

Jankeje K, Amiri M, Albani JR. Relation between Human Serum Albumin Structure and Fluorescence Decay Parameters of Tryptophan Residue 214. Encycl Anal 
Chem. 2017;1-19.

24. Hidrovo CH, Hart DP. Emission reabsorption laser induced fluorescence (ERLIF) film thickness measurement. Meas Sci Technol. 2001;12(4):467-77.

25. Huang $\mathrm{X}, \mathrm{Xu} \mathrm{D}$, Chen J, Liu J, Li Y, Song J, et al Smartphone-based analytical biosensors. 2019;5339-51.

26. Tran M V., Susumu K, Medintz IL, Algar WR. Supraparticle Assemblies of Magnetic Nanoparticles and Quantum Dots for Selective Cell Isolation and Counting on a Smartphone-Based Imaging Platform. Anal Chem. 2019;91(18):11963-71.
27. Nelson N, Sander D, Dandin M, Prakash SB, Sarje A, Abshire P. Handheld fluorometers for lab-on-a-chip applications. IEEE Trans Biomed Circuits Syst. 2009;3(2):97-107.

28. Pijanowska DG, Kossakowska A, Torbicz W. Electroconductive polymers in (Bio)chemical sensors. Biocybern Biomed Eng. 2011;31(4):43-57.

29. Yang M, Zhang Y, Cui M, Tian Y, Zhang S, Peng K, et al. A smartphone-based quantitative detection platform of mycotoxins based on multiple-color upconversion nanoparticles. Nanoscale. 2018;10(33):15865-74 
